\title{
Acumulación de plomo en hueso y sus efectos en la salud
}

Luz Helena Sanín, M.D., D.Sc., ${ }^{(1,2)}$ Teresa González-C ossío, Ph.D., ${ }^{(1)}$ Isabelle Romieu, M.D., D.Sc., ${ }^{(3)}$

Mauricio Hernández-Avila, M.D., D.Sc.(1)

\section{Sanín LH, González-Cossío T, Romieu I, Hernández-Avila $M$. Acumulación de plomo en hueso y sus efectos en la salud. Salud Publica Mex 1998;40:359-368.}

\section{Resumen}

Los efectos nocivos del plomo han sido conocidos desde tiempos antiguos por su amplia gama; este metal afecta prácticamente todos los órganos y sistemas del cuerpo humano. Recientemente se han documentado efectos tóxicos crónicos en los sistemas cardiovascular y nervioso, con niveles que antes se consideraban seguros. El sitio primario de almacenamiento de plomo en el organismo es el hueso, pero sólo hasta fechas recientes se ha clarificado la significancia toxicológica de este hecho. En este artículo se analiza el papel del plomo en hueso como fuente de exposición endógena, como biomarcador de exposición crónica y como tejido blanco. Se presentan además los avances recientes para la medición de plomo en hueso mediante los rayos Xfluorescentes (XRF). Finalmente se revisa la importancia, para la salud pública, de estudiar y medir el plomo en hueso en lugares con una historia de exposición crónica como la Ciudad de México y en los ambientes ocupacionales, especialmente como fuente remanente de exposición. Se analiza especialmente el caso de mujeres en edad reproductiva, quienes pueden convertirse en fuentes de exposición para el feto y para el niño amamantado.

Palabras clave: plomo/marcadores biológicos; exposición ocupacional; exposición materna

\section{Sanín LH, González-Cossío T,}

Romieu I, Hernández-Avila $M$.

Lead storage in bone

and its effects on health.

Salud Publica Mex 1998;40:359-368.

\begin{abstract}
A bstract
The adverse effects of lead have been known for long since the metal affects practically all organs and systems of the human body. Recently, toxic effects have been reported in the cardiovascular and nervo us systems at lead levels previo usly considered to be secure. The main lead storage site in the body is bone. The toxicologic significance of this fact has been clarified only recently. The present study analyzes the role of lead as an endogenous source of exposure, as a chronic exposure biomarker and as a target organ. Recent advances to measure bone lead through fluo rescent $X$-Rays are discussed. Additionally, the importance of bone lead from a public health perspective in places with a chronic history of exposure such as Mexico City, and in some occupational environments is reviewed with particular attention placed on reproductive age women, who are potential lead sources for the fetus and lactating infant.
\end{abstract}

Key words: lead/biological markers; occupational exposure; maternal exposure

(1) Centro de Investigación en Salud Poblacional, Instituto N acional de Salud Pública, México.

(2) Universidad Autónoma de Chihuahua, Chihuahua, México.

(3) Centro Panamericano de Ecología Humana y Salud, Metepec, Estado de México, México.

Fecha de recibido: 16 de julio de 1997 • Fecha de aprobado: 27 de abril de 1998

Solicitud de sobretiros: Dra. Luz Helena Sanín A guirre. Centro de Investigación en Sistemas de Salud, Instituto N acional de Salud Pública. Av. Universidad 655, colonia Santa María A huacatitlán, 62508 Cuernavaca, Morelos, México. 
D esde tiempos muy antiguos se conocen los efectos nocivos del plomo en el organismo humano; sin embargo, sólo desde hace unas décadas y utilizando una metodología más avanzada, ${ }^{1-9}$ se han detectado los daños que ocasiona el plomo en niveles bajos. En investigaciones llevadas a cabo recientemente se han destacado los peligros potenciales que representan las fuentes endógenas de plomo (depósitos óseos), especialmente en poblaciones con una historia de exposición crónica y, por consiguiente, con gran riesgo de movilización de metales tóxicos almacenados en el hueso en situaciones fisiopatológicas que aumentan la resorción ósea. ${ }^{10-15}$

El plomo tiene efectos tóxicos en muchos órganos, sistemas y procesos fisiológicos, incluyendo el desarrollo de la línea roja hemática, los riñones, el sistema cardiovascular, el aparato reproductor y, problamente el aspecto de mayor gravedad, el desarrollo del sistema nervioso central. La naturaleza de las manifestaciones de toxicidad depende no sólo de la magnitud de la exposición sino también de las características de la persona expuesta; la neurotoxicidad del plomo es más crítica para el feto en desarrollo y el niño en crecimiento que para los adultos.

Diversos investigadores mexicanos ${ }^{2,3,16-22}$ han estudiado los efectos de la contaminación por plomo sobre los niveles sanguíneos de la población en la Ciudad de México. Si bien durante los últimos años se han establecido medidas destinadas a disminuir las concentraciones ambientales de este metal, apenas ha comenzado a llevarse a cabo la medición de las fuentes endógenas potenciales de contaminación (por la liberación de plomo almacenado en el tejido óseo), especialmente en las mujeres, así como de sus consecuencias funcionales.

Las acciones tóxicas del plomo se atribuyen a su afinidad por los sitios de acción molecular del calcio; el metal actúa como sustituto del calcio en varios eventos regulatorios intracelulares, ya que es capaz de activar las fosfodiesterasas dependientes de la calmodulina y las proteínas cinasas independientes de la misma, ${ }^{23}$ teniendo efectos, además, sobre los canales de calcio. Estas reacciones moleculares pueden contribuir a bajas dosis del plomo y a efectos sutiles sobre la función cerebral y la de otros sistemas y aparatos. El depósito de plomo en hueso está influido por prácticamente todos los procesos que afectan el depósito o la movilización del calcio en el mismo, aunque se aclara que, como toda analogía, ésta también tiene sus limitaciones ya que, por ejemplo, la distribución de ambos iones en tejido sanguíneo es muy diferente.

Cada día se reconoce más el significado toxicológico del plomo acumulado en hueso, ${ }^{10,15}$ si bien mu- chos de los mecanismos que ocasionan el daño aún no no están claramente caracterizados. En este trabajo se analizarán tres aspectos de interés tanto clínico como de salud pública respecto a la acumulación del plomo en el hueso: primero, en el hueso se realiza la medición más significativa de exposición acumulada al plomo; segundo, opera como reservorio de plomo, el cual puede ser movilizado en estados fisiológicos y patológicos en los que aumenta la resorción ósea (embarazo, lactancia, menopausia, inmovilidad, senectud, tirotoxicosis, etc.), causando efectos adversos en otros tejidos y atravesando la barrera placentaria con graves consecuencias para el feto, ${ }^{24,25}$ tercero, se ha reconocido que el esqueleto es un importante blanco de la toxicidad causada por el plomo, cuyos efectos incluyen perturbación del desarrollo óseo y de la formación y resorción óseas tal como lo ha planteado Silbergeld. ${ }^{10}$ Por otra parte, en este trabajo se discutirán las técnicas de medición de plomo en hueso que se usan en la actualidad y las perspectivas del estudio y la medición de plomo en hueso en términos de salud pública.

\section{Plomo en hueso como biomarcador}

En los últimos años se ha incrementado el interés por descubrir y validar marcadores biológicos que permitan conocer mejor los mecanismos de toxicidad del plomo. El Consejo Nacional de Investigación (NCR, por sus siglas en inglés) de Estados Unidos de América $(\mathrm{EUA})^{26}$ ha clasificado los marcadores biológicos en tres tipos: de exposición, de efecto y de susceptibilidad.

La medición de plomo en sangre completa ha sido, durante mucho tiempo, uno de los biomarcadores de dosis interna más utilizados por las bondades que presenta en cuanto a su obtención, análisis e interpretación. Sin embargo, éste sólo mide exposición reciente o constante (exógena y/o endógena), ya que su vida media es de 35 días. ${ }^{27,28}$ Otros biomarcadores sanguíneos como la protoporfirina eritrocítica y la inhibición de la ALA deshidratasa miden efecto y se correlacionan con concentraciones sanguíneas de plomo de 20-25 $\mu \mathrm{g} / \mathrm{dl}$; asimismo, con concentraciones menores a ésta se han documentado efectos también tóxicos. También se han notificado efectos crónicos, como las alteraciones conductuales y la hipertensión, en bajas concentraciones de plomo en sangre.

En 1972 Needleman y colaboradores ${ }^{29}$ utilizaron por primera vez la decidua dental para medir exposición acumulada al plomo en niños. Sin embargo, ese biomarcador no es práctico, ya que los dientes no están disponibles en todas las poblaciones o en todas las edades, y la acumulación del plomo en los mismos varía a lo largo de la vida. ${ }^{14}$ 
Por ser el principal compartimento mineral del cuerpo humano, el tejido óseo se ha convertido en un elemento importante en el estudio de la exposición acumulada a plomo. Los análisis químicos han revelado que, en los adultos, cerca de 95\% del plomo en el cuerpo se almacena en los huesos; en los niños la cifra se aproxima al $70 \%{ }^{30}$ El hueso es un tejido vivo, dinámico, y su proceso de formación y resorción está controlado por diferentes factores metabólicos y hormonales; además, su fisiología es muy compleja, ${ }^{30,31}$ de tal manera que los diferentes tipos de hueso tienen tasas de crecimiento y mineralización distintas y, por ende, acumulaciones de plomo variables. La vida media del plomo en hueso es de 5 a 19 años $^{32}$ y aumenta en el hueso cortical. En otras épocas el plomo en hueso podía medirse únicamente por biopsia; hoy en día la tecnología ofrece técnicas inocuas y relativamente confiables.

La concentración de plomo en hueso es un biomarcador de exposición, tanto de dosis interna como de dosis biológica efectiva para tejidos como el óseo. ${ }^{32,33}$ Por otra parte, es un indicador que refleja la exposición acumulada a plomo, a diferencia de la concentración en sangre completa que refleja la exposición ambiental reciente. Se ha sugerido, además, que el plomo en hueso tiene una relación más directa con los niveles de plomo plasmático que el nivel de plomo en sangre completa ${ }^{34}$ en las situaciones especiales que se asocian con un aumento en la resorción ósea. La asociación más intensa con plomo plasmático tiene un mejor significado biológico, ya que aquél constituye la fracción lábil y biológicamente activa del plomo capaz de cruzar, por ejemplo, la barrera placentaria y alcanzar los tejidos fetales.

Cake y colaboradore ${ }^{35}$ midieron plomo en hueso (trabecular y cortical), plomo sérico y plomo en sangre completa en 49 trabajadores expuestos a plomo. Encontraron una correlación estrecha entre las mediciones de plomo en plasma y en hueso. La correlación fue mayor entre los niveles de plomo en hueso trabecular y plomo plasmático. La proporción de plomo plasmático/ plomo en sangre completa varió de 0.8 a $2.5 \%$.

Estos hallazgos implican que los niveles de plomo en sangre completa, que son el biomarcador usual en la práctica clínica y epidemiológica, pueden no representar adecuadamente, en todos los casos, los niveles de plomo plasmático. En algunos casos el plomo plasmático puede estar más influido por los niveles de plomo en hueso, que pueden ser una fuente significativa de plomo plasmático, especialmente en las situaciones en las cuales la movilización se incrementa, como en los estados fisiológicos o patológicos que promueven la resorción ósea.
Como un ejemplo de la posibilidad de que el plomo en hueso correlacione mejor con el plasmático en ciertas situaciones fisiopatológicas y sea por ello un mejor biomarcador para algunos efectos, recientemente $\mathrm{Hu}$ y colaboradores ${ }^{34}$ encontraron una asociación negativa entre los niveles de plomo en hueso y la concentración de hemoglobina, a pesar de la presencia concomitante de bajos niveles de plomo sanguíneo. Watanabe y colaboradores ${ }^{36}$ encontraron correlaciones muy bajas entre los niveles de plomo en hueso y en sangre completa ( 0.10 con tibia y 0.17 con rótula) en un estudio realizado con carpinteros.

En los estudios recientes de Schütz y colaboradores $^{37}$ y de Hernández-Avila y colaboradores, ${ }^{38}$ se notifica una alta correlación entre plomo en sangre completa y plomo en plasma; Hernández-Avila encontró, además, una influencia independiente y adicional para los niveles de plomo en plasma, a partir de las concentraciones de plomo en hueso, especialmente trabecular (rótula), en niveles de exposición considerablemente más bajos que los informados previamente. Otro hallazgo de gran interés en ese trabajo fue que la relación plomo plasmático/plomo en sangre total varía significativamente con las concentraciones de plomo en hueso, mas no con las concentraciones en sangre total. Aún no se define plenamente la relación real entre estos dos biomarcadores. ${ }^{26}$

La concentración de plomo en hueso, utilizada como un biomarcador más sensible que el plomo en sangre completa, para efectos sugeridos a bajas dosis y con resultados contradictorios al usar otro tipo de biomarcadores, puede ayudar a definir la magnitud y el sentido de dichas asociaciones. Hasta ahora son pocos los estudios de efecto en los que se ha utilizado el plomo en hueso como biomarcador; uno de ellos es el que se llevó a cabo en la Ciudad de México, donde se encontró una relación negativa, estadísticamente significativa, entre la concentración de plomo en tibia materna y el peso al nacer. Se observó una diferencia media de $156 \mathrm{~g}$ en el peso al nacer, entre los hijos de madres pertenecientes al primero y cuarto cuartil de los niveles de plomo en tibia (nivel no detectable y $20.7 \mu \mathrm{g}$ $\mathrm{Pb} / \mathrm{g}$ hueso mineral respectivamente), después de ajustar por los predictores más importantes de peso al nacer. ${ }^{39}$ La media de plomo en hueso para este grupo de mujeres $(\mathrm{n}=272)$ fue de 9.8 y $14.2 \mu \mathrm{g} \mathrm{Pb} / \mathrm{g}$ hueso mineral para tibia y rótula, respectivamente. Otro ejemplo es un estudio realizado en Boston ${ }^{40}$ acerca de la relación entre plomo en hueso y antropometría en una muestra de 54 niños. Las medias fueron de 1.2 y $5.0 \mu \mathrm{g}$ $\mathrm{Pb} / \mathrm{g}$ de hueso mineral en tibia y rótula, respectivamente. No se encontró asociación de plomo en hueso y crecimiento físico. 
El plomo en hueso constituye una alternativa como biomarcador para efectos crónicos y para aquellos efectos respecto a los cuales surge controversia al utilizar otro tipo de biomarcadores; además, es un complemento del uso de plomo en sangre completa, el cual, aun con su problema de temporalidad, es más útil para vigilancia epidemiológica laboral y otro tipo de efectos. Se restringe su uso para fines de investigación, ya que hacerlo en forma generalizada o con fines clínicos no representa una alternativa viable en cuanto a la relación costo/beneficio.

\section{Plomo en hueso como fuente de exposición endógena}

Aunque desde hace muchos años se ha reconocido que el plomo se acumula en el hueso, se tenía la idea de que se trataba de un secuestro, de un depósito con una sola vía, en el cual se iba acumulando el plomo removido de la circulación y de los tejidos blandos. ${ }^{14}$ Se trata de un concepto totalmente incorrecto, ya que el depósito y la remoción del plomo en hueso sigue exactamente la activa fisiología del calcio que está sometida a los efectos de factores generales, tales como la nutrición y el ejercicio, y de factores específicos como las influencias hormonales y metabólicas esquematizadas en la figura 1. Entre los elementos que modifican la fisio- logía del plomo están los factores de crecimiento, las proteínas derivadas del hueso y otras señales fisiológicas como el 1,25-dihidroxicalciferol, los estrógenos, la hormona paratiroidea, la calcitonina, la hormona del crecimiento, la prolactina, la tirotropina y nutrimentos como el calcio, el zinc y el fósforo. ${ }^{14,31,41}$

La fisiología del hueso, como ya se mencionó, es compleja y por ello diferentes tipos de hueso tienen diferentes tasas de crecimiento y mineralización, así como diferente densidad final. ${ }^{30}$ Se conoce como hueso cortical a aquel que tiene predominantemente este tipo de tejido óseo (cortical) y cuyos ejes longitudinales coinciden con las líneas de mayor esfuerzo; este hueso es más denso y mineralizado. El hueso trabecular está compuesto mayoritariamente por tejido óseo de tipo esponjoso en el cual el hueso laminar se dispone formando trabéculas y, por ello, es menos denso que el cortical. La concentración y la vida media del plomo en hueso no parecen ser iguales en hueso trabecular y hueso cortical. ${ }^{42}$ Algunos trabajos experimentales sugieren que hay una mayor movilidad del plomo en hueso trabecular que en el cortical. ${ }^{27,43,44}$ Por otra parte hay evidencia de que el plomo óseo puede regresar a la sangre en proporciones sustanciales (45 a $70 \%$ del total de plomo en sangre completa), después de disminuir la exposición exógena ${ }^{45,46} \mathrm{o}$ en circunstancias patológicas o fisiológicas que implican mayor

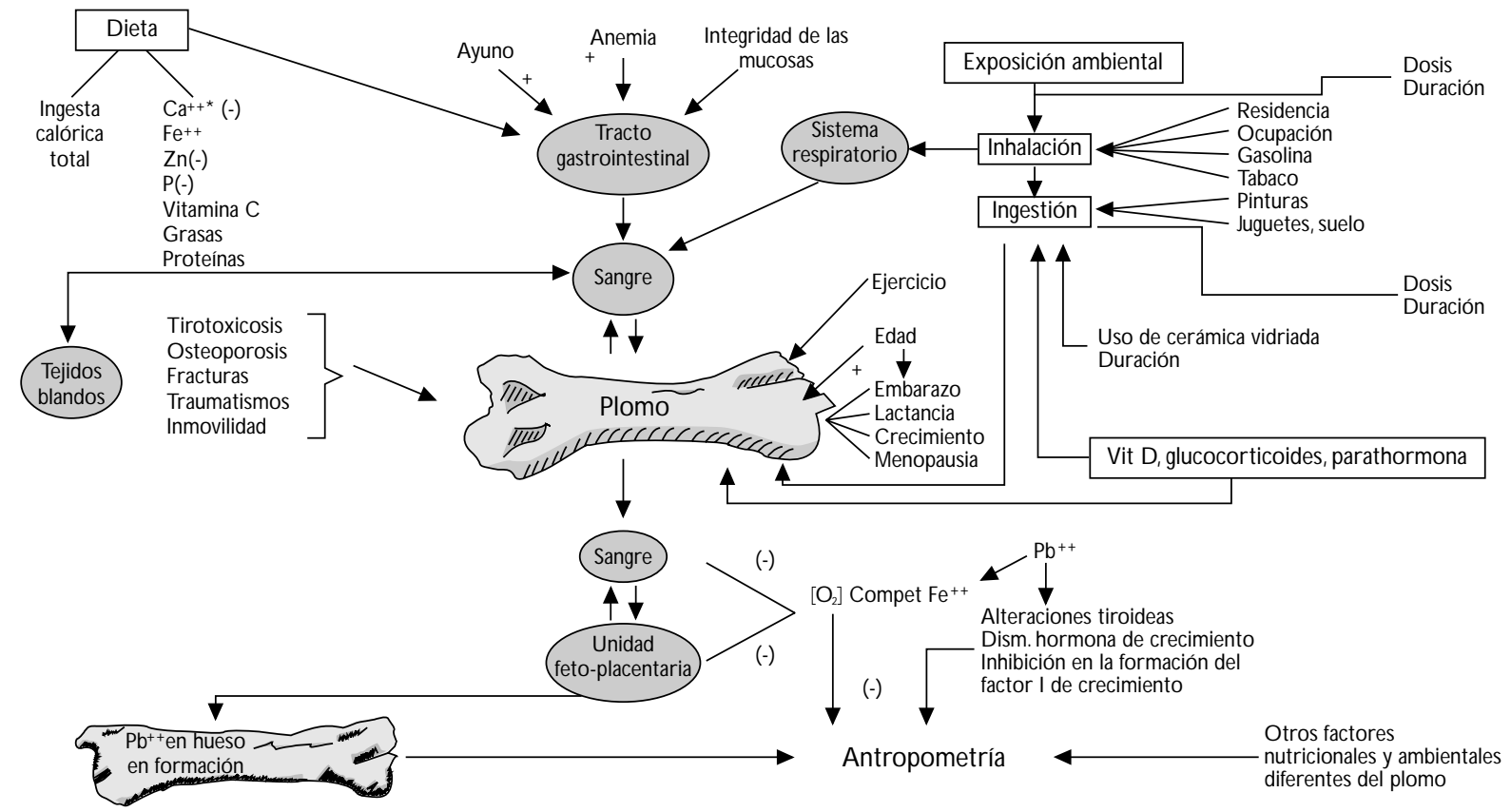

* Competencia en el nivel molecular

Figura 1. Modelo biológico del plomo en hueso 
resorción ósea. ${ }^{14,47,48}$ Los niveles sanguíneos pueden mantenerse altos o iguales a partir de los depósitos óseos, aun después de retirar la exposición aguda a plomo. En un estudio con 36 trabajadores expuestos laboralmente al plomo, 27 de ellos activos y 9 retirados, se encontró una correlación de 0.44 entre plomo en sangre y plomo en hueso en los activos, y de 0.93 entre los retirados. ${ }^{49}$

El trabajo con radioisótopos en animales ha demostrado que el plomo en hueso es una fuente endógena de exposición, ${ }^{46}$ y Gulson y colaboradores ${ }^{45}$ lo han documentado usando la misma técnica en migrantes europeos de países con altas concentraciones de plomo a Australia.

Se han notificado cambios mas drásticos en los niveles de plomo en sangre, a partir de los de hueso, en casos de menopausia, con un incremento de hasta $25 \%$ en los cinco años posteriores al evento, ${ }^{47}$ así como en niños después de fractura e inmovilidad ${ }^{50} \mathrm{y}$ en casos de tirotoxicosis. ${ }^{25}$

El embarazo implica una mayor demanda de calcio, tanto de la dieta como de los almacenamientos fisiológicos en tejido óseo. ${ }^{14}$ Estas demandas surgen de los requerimientos fetales para osificación y crecimiento, los cuales tienen su acmé durante el tercer trimestre del embarazo. El hueso materno funge como fuente de calcio en esa etapa. Se observan cambios en la tasa de formación y de resorción, especialmente en mujeres embarazadas con dietas deficientes en calcio. Esta movilización ósea estimula en gran medida la liberación de plomo, el cual atraviesa libremente la barrera placentaria, de tal forma que el plomo de hueso se convierte no sólo en fuente endógena para la madre sino también para el feto en desarrollo. ${ }^{14,31}$ Thompson ${ }^{51}$ informó en 1985 acerca de la existencia de manifestaciones clínicas de intoxicación aguda por plomo en una mujer embarazada y en su bebé, como consecuencia de la movilización del plomo almacenado en huesos.

$\mathrm{Al}$ estudiar el plomo en hueso como fuente de exposición endógena, es muy importante considerar los factores que pueden agravar la remoción ósea en los estados fisiopatológicos. Para las mujeres embarazadas, estas condiciones incluyen dieta, estado general de salud, edad y paridad, entre otras. ${ }^{14}$ Son pocos los trabajos que se han hecho sobre los predictores nutricionales y ambientales de plomo en hueso. En un estudio piloto realizado en la Ciudad de México en mujeres puérperas lactantes ${ }^{52}$ se encontró que los predictores significativos de los niveles de plomo en hueso trabecular (rótula) eran: años de residencia en la Ciudad de México; bajo consumo de alimentos ricos en calcio; ningún consumo de calcio durante el embarazo y, para el caso de hueso cortical (tibia), a estos predictores se agregaba la edad.

Rabinowitz ${ }^{28}$ estimó que para una vida media de actividad ósea de 35 años y una cantidad total de $200 \mathrm{mg}$ de plomo en hueso, este último podría liberar cada día $11 \mu \mathrm{g}$ de plomo a la sangre, de tal modo que el incremento observable en las concentraciones sanguíneas sería de $3 \mu \mathrm{g} / \mathrm{dl}$, cantidad que podría aumentar hasta $7 \mu \mathrm{g} / \mathrm{dl}$ en caso de envejecimiento $\mathrm{u}$ otros estados fisiopatológicos que provoquen desmineralización. En el caso de un trabajador expuesto a plomo que hubiese acumulado una carga total de $450 \mu \mathrm{g}$ y hubiese presentado un aumento en la tasa de desmineralización de $10 \%$ en un año, el plomo en hueso podría incrementar los niveles sanguíneos hasta $26 \mu \mathrm{g} / \mathrm{dl}$.

En otro estudio realizado en $\mathrm{EUA}^{53}$ para establecer los principales determinantes de plomo en hueso en un grupo de 719 varones en edad adulta, se encontró que la edad, el tabaquismo y la baja escolaridad eran los principales predictores, tanto para hueso cortical como trabecular. Este mismo estudio indica una diferencia de $4.3 \mu \mathrm{g} / \mathrm{dl}$ de plomo en sangre, entre el quintil más bajo y el más alto de plomo en rótula. Además, el factor que explicó la mayor parte de la varianza de plomo en sangre fue el nivel de plomo en rótula. Con este hallazgo los autores concluyeron que el plomo en hueso es la fuente principal de exposición en este grupo aun tratándose de un grupo con una baja exposición ambiental.

\section{El hueso como tejido blanco del plomo}

Se trata aquí de un aspecto que ha empezado a ser considerado a partir de hace muy poco tiempo: ${ }^{14}$ Pounds y colaboradores ${ }^{15}$ encontraron evidencias, tanto in vivo como in vitro, que sugieren que las células óseas pueden ser afectadas por la presencia de plomo. Cuando los animales son expuestos experimentalmente al plomo se reduce su crecimiento fetal y neonatal. ${ }^{54}$ por otra parte, la estatura baja ha sido desde 1920 uno de los criterios diagnósticos para la intoxicación crónica por plomo en niños australianos, según lo citado por Rothenberg y colaboradores. ${ }^{55}$

Las evidencias experimentales que permiten proponer algunos mecanismos fisiopatológicos probables para el establecimiento de una lesión ósea son: ${ }^{14}$

- Alteración del cristal de hidroxiapatita y, por consiguiente, alteración de la adhesión de la célula ósea a la matriz mineralizada.

- Competencia entre el plomo y el calcio en sus sitios de unión, con alteración de la homeostasis del calcio. 
- Alteración de la capacidad de las células óseas para responder a las hormonas.

- Daño a la capacidad de las células óseas para sintetizar y/o excretar componentes de la matriz (colágeno, sialoproteínas).

- Inhibición de la producción de osteocalcina por parte de los osteoblastos.

- Alteración en el acople funcional de osteoblastos y osteoclastos.

La evidencia de lesiones locales por la permanencia de proyectiles con plomo en el tejido óseo refuerza esta hipótesis. ${ }^{56}$ El esqueleto en desarrollo parece ser más sensible que el esqueleto adulto a la acción tóxica del plomo. Con deficiencia de calcio estos efectos son exacerbados y hay una osificación retardada en fetos expuestos al plomo. ${ }^{10}$

El crecimiento posnatal también parece ser sensible a niveles sanguíneos de plomo considerados como bajos $\left(10 \mu \mathrm{g} / \mathrm{dl}\right.$ o menos) ${ }^{55}$ En un análisis detallado de la segunda Encuesta Nacional de Salud y Nutrición (NHANES II, por sus siglas en inglés) en EUA que hicieron Schwartz y colaboradores ${ }^{57}$ se encontró una relación inversa entre niveles sanguíneos de plomo, por un lado, y la talla y la circunferencia torácica, por otro, en niños menores de siete años: cada incremento de $10 \mu \mathrm{g} / \mathrm{dl}$ de plomo en sangre se asoció con $2 \mathrm{~cm}$ de disminución en la talla. Esta asociación ha sido confirmada por estudios retrospectivos ${ }^{58} \mathrm{y}$ por resultados preliminares en 260 niños en un estudio prospectivo. ${ }^{59-61}$ Bellinger y colaboradores ${ }^{62}$ también informaron acerca de una asociación negativa entre talla y plomo en sangre más allá de los $15 \mu \mathrm{g} / \mathrm{dl}$. Frisancho y Ryan encontraron una asociación inversa entre la estatura y la concentración de plomo en sangre en niños de origen mexicano, residentes en EUA, utilizando datos de la NHANES II. ${ }^{63}$

En algunos estudios recientes ${ }^{10,15}$ se han encontrado bajos niveles plasmáticos de osteocalcina (una proteína de la matriz ósea sintetizada por los osteoblastos) en niños intoxicados por plomo. A pesar de algunos errores metodológicos (falta de control de factores confusores o falta de poder estadístico), en estos estudios se observa un deterioro en el crecimiento posnatal cuando existen niveles de plomo en sangre por debajo de los $10 \mu \mathrm{g} / \mathrm{dl}$. En estudios experimentales se ha encontrado una asociación negativa entre los niveles de plomo y la talla y se ha probado la reversibilidad de este efecto ${ }^{64}$ También hay evidencia experimental de que el hueso es un tejido blanco de toxicidad del plomo: en animales de experimentación Pounds y colaboradores ${ }^{15}$ notificaron una asociación entre el plomo en sangre y la incidencia de malformaciones esqueléticas, si bien en otras investigaciones no se ha podido demostrar esta asociación. ${ }^{65,66}$

Se han propuesto al menos tres mecanismos adicionales a la propia alteración ósea para explicar cómo pueden afectar el crecimient $0^{57}$ los bajos niveles de plomo. Estos mecanismos incluyen: a) interferencia del plomo con reacciones mediadas por calcio como segundo mensajero; b) disminución de enzimas hemodependientes, ya sea por el plomo o por deficiencia de hierro, y c) toxicidad neuroendócrina posiblemente relacionada con la inhibición de receptores en hipotálamo. Se ha sugerido, respecto a este último punto, que la alteración en el crecimiento puede deberse a la secreción disminuida de la hormona de crecimiento, misma que genera una reducción en la producción del factor I de crecimiento, o a que el plomo inhiba directamente la secreción de este último factor. ${ }^{26,40,67} \mathrm{Sin}$ embargo, la lesión directa del plomo sobre el hueso en crecimiento parece convertirse en una hipótesis cada vez más plausible.

\section{Medición de plomo en hueso}

Todas las consideraciones anteriores sobre las bondades de la medición de plomo en hueso podrían ser meramente académicas si no fuera porque los avances en la instrumentación han permitido la medición in vivo del plomo óseo, usando la técnica de rayos-X fluorescentes $(\mathrm{XRF})$, la cual ofrece ventajas sobre otras técnicas para estimación de plomo en hueso, como la quelación con ácido etilen-diamino-tetracético (EDTA). ${ }^{68-70}$

En 1972 Ahlgren y Mattson ${ }^{35}$ realizaron las primeras mediciones de plomo en hueso in vivo, utilizando como fuente de poder, rayos $\gamma$ provenientes de ${ }^{57} \mathrm{Co}$; desde entonces la técnica se ha modificado sustancialmente.

El principio de esta inocua técnica de XRF es la utilización de una radiación gamma de bajo nivel para provocar la emisión de fotones fluorescentes del área anatómica de interés. Los fotones son detectados y caracterizados, según su longitud de onda, mediante un programa de computadora diseñado especialmente (figura 2). La técnica no es invasiva, es indolora y requiere de muy poca exposición a la radiación, pues se considera equivalente a una exposición de diez minutos al sol y a menos de $10 \%$ de una radiografía de tórax. ${ }^{44,71-77}$ Básicamente existen dos técnicas de XRF: la $\mathrm{L}-\mathrm{XRF}$, que se concentra en la emisión de electrones del orbital L, y la K-XRF cuya radiación penetra en el hueso más profundamente y se concentra en las emisiones del orbital K.

Cada técnica proporciona una información particular pues detecta diferentes tipos de depósitos de plomo en hueso. Así, la L-XRF parece identificar un 


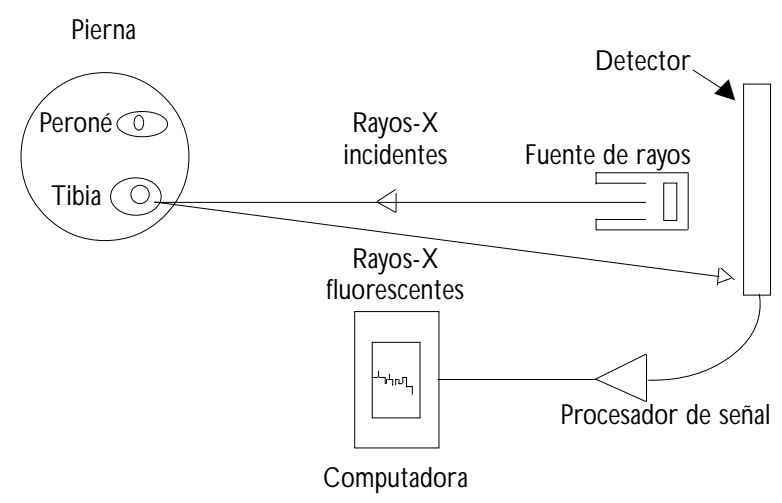

Fuente: Referencia 11

Figura 2. Diagrama del sistema de detección de la FLUORESCENCIA PARA MEDICIONES DE PLOMO EN HUESO

depósito de plomo que está inmediatamente por debajo del periostio. Este pico podría corresponder a plomo recientemente depositado y correlaciona con el metal que es secuestrable por el EDTA. Por el contrario, la K-XRF suministra una visión más amplia, ya que penetra a mayor profundidad, aproximadamente entre 20 y $40 \mathrm{~mm}$ dentro del hueso. ${ }^{30}$

La técnica K-XRF varía respecto al isótopo radiactivo utilizado, la geometría del arreglo entre fuente y detector, la programación utilizada, los aditamentos electrónicos y el sitio anatómico seleccionado para la medición.

El grupo de investigación de la Universidad de Harvard ha desarrollado un instrumento de K-XRF que utiliza ${ }^{109} \mathrm{Cd}$, como fuente de radiación para realizar mediciones en pierna. ${ }^{78-80} \mathrm{El}$ número de fotones fluorescentes de plomo se compara con el que llega de la hidroxiapatita, lo cual permite expresar la medición en microgramos de plomo, por gramo de hueso mineral $(\mu \mathrm{g} \mathrm{Pb} / \mathrm{g})$. Este aparato tiene, además, dos características que lo han hecho adaptable para estudios epidemiológicos: la facilidad de su manejo y la adaptación de un programa que permite estimar la imprecisión de cada medida, como si fuera una desviación estándar de múltiples mediciones. Esto último permite hacer ajustes en los resultados finales.

La variabilidad inter e intrahueso es minimizada al tomar mediciones en hueso cortical y trabecular y realizar las mediciones en el mismo sitio. ${ }^{77}$ Se ha puesto a prueba la exactitud del método con estudios llevados a cabo en cadáveres y que han mostrado una correlación de 0.98 con las mediciones químicas ${ }^{81}$

Actualmente existen en el mundo cerca de 12 grupos de trabajo que utilizan XRF, la mayoría de ellos en
EUA; en América Latina existe uno solo, ubicado en México.

\section{Perspectivas}

En México, desde 1682, se ha observado la contaminación ambiental por plomo y sus potenciales efectos adversos. ${ }^{82}$ La cantidad de plomo liberada al ambiente está estrechamente relacionada con la producción del metal en los últimos 5000 años; aproximadamente la mitad del plomo que se produce se libera como contaminante, ${ }^{26}$ así, la producción anual mundial estimada es de cerca de 3.4 millones de toneladas métricas, de las cuales se liberan al ambiente cerca de 1.6 millones. El plomo es indestructible y no puede ser transformado en una forma inocua. La dispersión del metal no conoce límites geográficos y contamina áreas lejanas al sitio de emisión original. En México, la producción anual de plomo es de 225472 toneladas. $^{83}$

El plomo que se encuentra en la atmósfera procede, básicamente, de la combustión de la gasolina con plomo; otras fuentes consideradas son el carbón, la combustión de aceite, la minería, la producción de cierto tipo de manufacturas, los fertilizantes, la incineración, la producción de cementos y la combustión de madera. Los efectos históricos ambientales de estas emisiones sobre los niveles de plomo en el humano han sido demostrados al comparar las concentraciones de este metal en restos de indígenas precolombinos y en el humano actual, de modo que se han encontrado niveles entre 100 y 1000 veces mayores para este último. ${ }^{26}$

En la Ciudad de México se ha estimado que anualmente se depositan en el ambiente hasta 15000 toneladas métricas de plomo, provenientes de la combustión de gasolina con plomo, situación prevaleciente al menos hasta $1990 .^{84}$

Si bien se han tomado medidas para reducir la concentración de plomo en las gasolinas, las pinturas, los alimentos enlatados y los juguetes, hoy en día persiste la tradición de utilizar cerámica vidriada; a lo anterior se aúna el plomo liberado al ambiente en años anteriores. ${ }^{85}$

La cerámica vidriada a baja temperatura libera cantidades sustanciales de plomo al contacto con alimentos, especialmente si éstos tienen un $\mathrm{pH}$ bajo. Hace poco tiempo se aprobó el uso de una nueva tecnología que incorpora productos derivados del boro, en el proceso de vidriado, en sustitución de la greta tradicional que contiene plomo. Empero, el uso de esta nueva técnica aún no se ha generalizado y tampoco se han evaluado sus resultados, de tal manera que se desconoce si el problema puede solucionarse en el corto pla- 
zo. Por otra parte, existen otras medidas que, si bien se han puesto en marcha y para las cuales existen normas, su cumplimiento no está sujeto a vigilancia.

Una persona residente en la Ciudad de México puede haber acumulado grandes cantidades de plomo en hueso, situación que adquiere mayor relevancia en el caso de las mujeres, al convertirse dichas reservas en fuentes potenciales de contaminación endógena, especialmente in utero. Esto es especialmente grave en el caso de la exposición ocupacional femenina al plomo y debe ser considerado seriamente en el establecimiento de las normas necesarias al respecto.

$\mathrm{Si}$ bien las altas concentraciones de plomo en sangre han disminuido en la población general, ${ }^{86,87}$ los efectos a bajas dosis merecen especial cuidado. Por un lado está el efecto negativo en la antropometría al nacer, cuya importancia reside en su relación con la morbilidad y la mortalidad, el crecimiento y el desarrollo. ${ }^{88}$ El efecto del plomo sobre el hueso en formación afecta la longitud y puede afectar asimismo el peso y el perímetro cefálico al nacer, indicadores de conocida asociación con la morbilidad y la mortalidad infantiles. Si a ello se agregan los efectos causados por las deficiencias de la nutrición, de gran prevalencia en México, ${ }^{89}$ el resultado es un problema sumamente importante desde el punto de vista de la salud pública.

No existen cifras que permitan estimar los niveles de plomo para la población global de la Ciudad de México; sin embargo, los datos emanados del Programa de Vigilancia Epidemiológica del Binomio MadreHijo que se llevó a cabo en nueve hospitales de esa ciudad ${ }^{90}$ permitieron estimar que, para 1993 , hasta $48 \%$ de las madres y $44 \%$ de los niños (sangre de cordón) incluidos en dicho programa tenían niveles de plomo sanguíneo por arriba de los $10 \mu \mathrm{g} / \mathrm{dl}$. Ese es el límite de seguridad que ha fijado el Centro para la Prevención y el Control de las Enfermedades de EUA, ${ }^{16,26,91}$ lo cual implica que un número considerable de niños nacidos en el Distrito Federal está en riesgo de sufrir los efectos tóxicos del plomo. Si bien para los niveles de plomo en hueso, no hay un límite de seguridad establecido, los valores que se han encontrado en la Ciudad de México son más altos que los notificados en otras partes del mundo. ${ }^{39}$

La magnitud de la contaminación por plomo en ciudades como la de México lleva a considerar que aun en el caso ideal de que se abatiera completamente la contaminación por fuentes exógenas, habría que enfrentar durante varias décadas los efectos negativos de las fuentes endógenas. En este sentido es particularmente grave la situación de los niños: el plomo afecta su peso al nacer y su desarrollo neuroconductual, y dicho efecto puede llegar a ser severo y prolongarse más allá de la infancia. ${ }^{26,67} \mathrm{Al}$ considerar lo anterior es fácil explicarse la relevancia que tiene el estudio de los predictores de plomo en hueso y su ensayo como un biomarcador más sensible para efectos sutiles.

La investigación debe tender, en la actualidad, a la intervención con miras a la eliminación. Las estrategias nutricionales pueden llegar a constituir la alternativa más viable y menos costosa para el control de las fuentes endógenas; entre éstas están la suplementación con calcio, hierro, zinc, etcétera. ${ }^{52}$

Es necesario mantener la vigilancia sobre los grupos más vulnerables como el binomio madre-hijo y la población ocupacionalmente expuesta, en particular la femenina, pero, además, es necesario vigilar el cumplimiento de las normas que se establecen.

La utilización de este biomarcador en poblaciones expuestas ocupacionalmente, tanto en grandes centros como en ciudades pequeñas, puede ayudar a dilucidar asociaciones controvertidas. Se sugiere, sin embargo, utilizarlo con fines de investigación en los lugares donde logísticamente sea más propicio y correlacionar los resultados con niveles de plomo en sangre completa, más fáciles de medir y mas útiles para la vigilancia epidemiológica.

El estudio y la utilización de la medición de plomo en hueso no sólo permitirán avanzar en el conocimiento de los efectos tóxicos crónicos y a bajas dosis de este metal, sino también descubrir posibles intervenciones.

\section{Referencias}

1. Landrigan P.Toxicity of lead at low dose. Br J Ind Med 1989;46:593-696. 2. Palazuelos $E$, Hernández $M$, Romieu I, Cortés $M$, Ríos $C$, Muñoz I. La contaminación por plomo en México. En: Restrepo I, ed. La contaminación atmosférica en México. Sus causas y sus efectos en la Salud. México, D.F.: Comisión N acional de Derechos Humanos. 1992;23-27.

3. Jiménez $C$, Romieu I, Palazuelos $E$, Muñoz I, Cortés $M$, Rivero $E$ et al. Factores de exposición ambiental y concentraciones de plomo en sangre en niños de la Ciudad de México. Salud Publica Mex 1993;35:599-606.

4. Ernhart CB, Wolf AW, Kennard MJ, Erhard P, Filipovich HF, Sokol RJ. Intrauterine exposure to low levels of lead:The status of the neonate. Arch Environ Health 1986; 41(5):2876-2891.

5. Ling $W$ et al. Study of lead absortion and its effect on children's development. Biomed Environ Sci 1989;2:325-330.

6. Schwartz J. Low-level lead exposure and children IQ : A meta-analysis and search for a threshold. Environ Res 1994;65:42-55.

7. Laraque D, McC omick M, N orman M et al. Blood lead, calcium status, and behavior in preschool children. Am J D is Child 1990; 144:186-189.

8. Schwartz J. Societal benefits of reducing lead exposure. Environ Res 1994;66:105-124

9. West W L, Knight EM, Edwards CH et al. Maternal low level lead and pregnancy outcomes. J N utr 1994;suppl 6:981s-986s. 
10. Silbergeld E, Sauk J, Somerman M, Todd A, MacN eill F, Fowler B et al. Lead in bone: Storage site, exposure source and target organ. N eurotoxicology 1993;14(2-3): 225-236.

11. Hu H, Milder F, Burger D. X-Ray flourescence: Issues surrounding the application of a new tool for measuring lead burden. Environ Res 1989; 49:295-317.

12. Hu H, Milder $M$, Burger DE. The use of $K-X$-Ray flourescence for measuring lead burden in epidemiological studies: $H$ igh and low lead burdens, and measurement uncertainty. Environ Health Perspect 1991;94: 107-110.

13. Rosen JF, Markowitz ME, Bijur ST, Jenks ST, W ielopolski L, Kalef-Ezra $J A$ et al. Sequential measurements of bone lead content by L-X -Ray flourescence in C aN a2-EDTA-treated lead-toxic children. Environ Health Perspect 1991;93:271-277.

14. Silbergeld EK. Lead in bone: Implications for toxicology during pregnancy and lactation. Environ Health Perspect 1991;91:63-70.

15. Pounds JG, Long GJ, Rosen JF. C ellular and molecular toxicity of lead in bone. Environ Health Perspect 1991:91:17-32.

16. Romieu I, Palazuelos E, Hernández-Avila M, Ríos C, Muñoz I, Jiménez C. Sources of lead exposure in Mexico City. Environ Health Perspect 1994;102:384-389.

17. Hernández M, Romieu L, Ríos C, Rivero A, Palazuelos E. Lead glazed ceramics as major determinants of blood lead levels in Mexican women. Environ Health Perspect 1991:94:117-120.

18. Romieu I, Palazuelos E, Meneses F, Hernández M.Vehicular traffic as a determinant of blood lead levels in children: A pilot study in Mexico City. Arch Environ Health 1992;47:246-249.

19. Muñoz H, Romieu I, Palazuelos E, Meneses F, Mancilla-Sánchez T, Meneses-González et al. Blood lead level and neurobehavioral development among children living in Mexico City. Arch Environ Health 1993;48(3):132-139.

20. Rothenberg SJ, Pérez IA, Perroni E, Schnaas L, C ansino S, Suro D et al. Fuentes de plomo en embarazadas de la Cuenca de México. Salud Pública Mex 1990;32:632-643.

21. Lara-Flores E,Alagon-C ano J, Bobadilla JL, H ernández-Prado B, C iscomani-Begoña $A$. Factores asociados a los niveles de plomo en sangre en residentes de la Ciudad de México. Salud Publica Mex 1989;31(5): 625-633.

22. Rothenberg S), Karchmer S, Schanaas L, Perroni E, Zea F, Fernández J. Changes in serial blood lead levels during pregnancy. Environ Health Perspect 1994;102:876-880

23. Goldstein GW. Evidence that lead acts as a calcium substitute in second messenger metabolism. N eurotoxicology 1993;14(2-3):97-101.

24. Goyer RA. Transplacental transport of lead. Environ Health Perspect 1990;89:101-105

25. Goldman RH, W hite R, Kales $S$, Hu H. Lead poisoning from mobilization of bone stores during thyrotoxicosis. Am I Ind Med 1993;24:1-8. 26. $N$ ational Research Council, C ommittee on Measuring Lead in C ritical Populations, Board on Environmental Studies and Toxicology, Commission on Life Sciences. Measuring lead exposure in infant, children, and other sensitive population. W ashington D.C.:N ational Academy Press, 1993.

27. Rabinowitz MB, W etherill GW, Kopple JD. Kinetic analysis of lead metabolism in healthy humans.J C lin Invest 1976;58:260-270.

28. Rabinowitz MB.Toxicokinetics of bone lead. Environ Health Perspect 1991;91:33-37.

29. N eedleman HL, Tuncay C, Shapiro I. Lead levels in deciduous teeth of urban and suburban American children. N ature 1972;235:111-112. 30. N ordberg GF, Mahaffey KR, Fowler BA. Introduction and summary. International workshop on lead in bone: Implications for dosimetry and toxicology. Environ Health Perspect 1991;91:3-7.
31. Marks SC, Popoff SN. Bone cell biology: The regulation of development, structure and function in the skeleton. Am J Anat 1988;183:1-44. 32. Gerhardsson L, Attewell R, Chettle DR, Englyst V, Lundström NG, $N$ ordberg GF et al. In vivo measurements of lead in bone in long-term exposed lead smelters workers. Arch Environ Health 1993;48:147-156. 33. Schulte PA, Perera FP. Molecular epidemiology. Principles and practices. San Diego (CA): A cademic Press, 1993.

34. $\mathrm{Hu} \mathrm{H}, \mathrm{W}$ atanabe $\mathrm{H}$, Payton M, Korrick KS, Rotnitzky A. The relationship between bone lead and hemoglobin. JAMA 1994;272:1512-1517.

35. Cake $\mathrm{Km}$, Bowins RJ,Vaillancourt C, G ordon CL, MacN utt RH, Laporte $R$ et al. The partition of circulating lead between serum and red cells is different for internal and external sources of lead. Am J Ind Med 1996:29:440-445

36. W atanabe $\mathrm{H}, \mathrm{Hu} \mathrm{H}$, Rotnitzky A. Correlates of bone and blood lead levels in carpenters. Am J Ind Med 1994;26:255-264.

37. Schütz A, Pergdahl IA, Kholm A, Skerfuing S. Measurement by IC P-MS of lead in plasma and whole blood of lead in workers and controls. 0 ccup Environ Med 1996;53:736-740.

38. Hernández-A vila M, Smith D, Meneses F, Sanín LH, Hu H. Lead kinetics in environmentally-exposed adults: the influence of bone and blood on plasma lead levels. Environ Health Perspect 1998. En prensa.

39. Gonzalez-C ossío T, Peterson K, Sanín LH, Fishbein E, Palazuelos E, Aro $A$ et al. Decrease in birth weight in relation to maternal bone lead burden. Pediatrics 1997;100:854-862.

40. Kim R, Hu H, Rotnitzky A, Bellinger D, N eedleman H. Chronic lead exposure and physical growth parameters in a 13-year follow-up study. Environ Health Perspect 1995;103:952-957.

41. Sauk JJ, Somerman MJ. Physiology of bone: Mineral compartment proteins as candidates for environmental perturbation by lead. Environ $\mathrm{H}$ ealth Perspect 1991:91:9-16.

42. Rosen JF, Crocetti AF, Balbi K, Balbi J, Bailey Ch, Clemente I et al. Bone lead content assessed by L-line $\mathrm{X}$-ray fluorescence in lead exposed and non-lead exposed suburban populations in the United States. Proc $N$ atl A cad Sci 1993:90:2789-2792.

43. Armstrong JE, Riihimäki V, Chettle DR, Paakkari A, Scott M, Somervaille $L$ et al. In vivo measurements of lead in bone at four anatomical sites: Long term occupational and consequent endogenous exposure. $\mathrm{Br} J$ Ind Med 1992:49:631-644.

44. W ittmers LE, W allgren J, A lich A, A ufderheide AC, Rapp G. Lead in bone. IV. Distribution of lead in human skeleton. Arch Environ Health 1988:43(6):381-390

45. Gulson BL, Mahaffey KR, Mizon KJ, Korsch MJ, Cameron MA,Vimpani G. C ontribution of tissue lead to blood lead in adult female subjects based on stable lead isotope methods.J Lab Clin Med 1995;125:703-712.

46. Smith D, 0 sterloh JD, Russell A. U se of endogenous, stable isotopes to determine release of lead from the skeleton. Environ Health Perspect 1996:104:60-66

47. Silbergeld EK, Schwartz J, Mahaffey K. Lead and osteoporosis; mobilization of lead from bone in postmenopausal women. Environ Res 1988:47:79-94.

48. Symanski E, Hertz-Picciotto I. Blood lead levels in relation to menopause, smoking, and pregnancy history. Am J Epidemiol 1995;141:10471058.

49.- Schütz A, Skerfing S, Mattson S, C hristoffersson J,A Algren L. Lead in vertebral bone biopsies from active and retired lead workers. Arch Environ Health 1987;42:340-346.

50.- Markowitz MR, W einberger HL. Immobilization-related lead toxicity in previously lead-exposed children. Pediatrics 1990;86:455-457.

51. Thompson GN, Robertson EF, Fitzgerald S. Lead mobilization during pregnancy. Med J Aust 1985;143:131. 
52. Hernández-Avila M, Gonzalez-Cossío T, Palazuelos E, Romieu I, Aro $A$, Fishbein $E$ et al. D ietary and environmental determinants of blood and bone lead levels in lactating post-partum women living in Mexico City. Environ Health Perspect 1996;104:1076-1082.

53. Hu H, Payton M, Korrick S, Aro A, Sparrow D, Weiss ST et al. Determinants of bone and blood lead levels among community-exposed middle-aged to elderly men. The normative aging study. Am J Epidemiol 1996;144:749-759.

54. Hammond PB, Minnema DJ, Shukla R. Lead exposure lowers the set point for food consumption and growth in weanling rats. Toxicol Appl Pharmacol 1990;160:80-87.

55. Rothenberg SJ, Schnaas-A rrieta L, Pérez-G uerrero IA, Perroni Hernández E, Mercado-Torres L, Gómez-Ruiz F et al.Prenatal and postnatal blood lead level and head circumference in children to three years: preliminary results from the México City prospective lead study.J ExpAn and Environ Epid 1993;3(1):165-172.

56. Slavin RE, Swedo J, Cartwright J,Viegas S, Custer EM. Lead arthritis and lead poisoning following bullet wounds: A clinicopathological, ultrastructural and microanalytic study of two cases. Hum Pathol 1988;19:223235.

57. Schwartz J, Angle CR, Pirkle JL, Pitcher H. Relation between childhood blood-lead levels and stature. Pediatrics 1986;77:281-288.

58. Lauwers MC, Hauspie RC, Susanne C, Verheyden J. Comparison of biometric data of children with high and low levels of lead in the blood. Am J Phys Antropol 1986; 69:107-116.

59. Shukla R, Bornschein RL, Dietrich KN, Mitchell T, Grote S, Berger 0 et al. Effects of fetal and early postnatal lead exposure on child's growth in stature. The Cincinnati lead study. En: Lindber SE, Hugchison TC, ed. International Conference. Heavy metals in the environment, Edimburgo, Escocia: CEP Consultants, 1987:210-212.

60. Shukla R, Bornschein RL, Dietrich KN, Buncher CR, Berger O, Hammond $P B$ et al. Fetal and infant lead exposure: Effects on growth in stature. Pediatrics 1989;84(4):604-612.

61. Shukla R, Dietrich KN, Bornschein R, Berger 0 , Hammond PB. Lead exposure and growth in the early preschool child: A follow-up report from the Cincinnati lead study. Pediatrics 1991;88:886-892.

62. Bellinger D, Leviton A, Rabinowitz M, Allred E, N eedleman H, Schoenbaum S.W eight gain and maturity in fetuses exposed to low levels of lead. Environ Res 1991:54:151-158.

63. Frisancho AR, Ryan AS. D ecreased stature associated with moderate blood lead concentrations in Mexican-A merican children. Am J C lin N utr 1991;54:516-519.

64. Hammond PB, Minnema DJ, Succop PA. Reversibility of lead induced depression of growth.Toxicol Appl Pharmacol 1993;123:9-15.

65. Sachs HK, Moel DI. Height and weight following lead poisoning in childhood. Am J Dis Child 1989;143:820-822.

66. Greene T, Ernhart CB. Prenatal and preschool age lead exposure: Relation with size. N eurotoxicol Teratol 1991;13:417-427.

67. N eedleman HL, Bellinger D.The health effects of low level exposure to lead. Annu Rev Public Health 1991;12:111-140.

68.Todd AC, MCN eill FE, Fowler BA. In vivo X-Ray fluorescence of lead in bone. Environ Res 1992;59:326-335.

69. Chettle DR, Scott MC, Somervaille LJ. Lead in bone: Sampling an quantitation using $\mathrm{K}-\mathrm{X}$-rays excited by $109 \mathrm{Cd}$. Environ Health Perspect 1991:91:49-55

70. Thomas BJ. Equipment desing issues for the in vivo $X$-Ray fluorescence analysis of bone lead. Environ Health Perspect 1991;91:39-43.

71.A ufderheide AC, W ittmers LE. Selected aspects of the spatial distribution of lead in bone. N eurotoxicology 1992;13:809-820.

72. Hu H, Tosteson T, Aufderheide AC et al. Distribution of lead in human bone. Atomic absorption measurement. En:Yasumura, ed. Advances in vivo body composition studies. N ueva York: Plenum Press, 1990.
73. Marcus AH. Multicompartment kinetic models for lead. Bone diffusion models for long-term retention. Environ Res 1985;36:441-458.

74. 0 'Flaherty E. Physiological based models for bone-seeking elements, lead absortion and disposition in childhood. Toxicol Appl Pharmacol 1995;131:297-308.

75. Landrigan PJ,Todd AC. Direct measurement of lead in bone.A promising biomarker. JAMA 1994;271(3):239-240.

76. Landrigan PJ. Strategies for epidemiological studies of lead in bone in occupationally exposed populations. Environ Health Perspect 1991;91: 81-86.

77. Hu H, Aro A, Rotnitzky A. Bone lead measured by X-Ray fluorescence: Epidemiological methods. Environ Health Perspect 1995;103 suppl 1: 105-110.

78. Kim R, Aro A, Rotnizky A, Amarasiriwadena Ch, Hu H. K X-Ray fluorescence measurements of bone lead concentration:The analysis of lowlevel data. Phys Med Biol 1995;40:1475-1485.

79. Hoppin JA, Aro A, W illiams PI, Hu H, Ryan PB. Validation of K-XRF bone lead measurement in young adults. Environ $H$ ealth Perspect 1995;103:78-83.

80. A ro A, Todd AC, A marasiriwadena $\mathrm{Ch}$ and $\mathrm{Hu} \mathrm{H}$. Improvements in the calibration of $109 \mathrm{C} \mathrm{d} \mathrm{K-X-Ray} \mathrm{fluorescence} \mathrm{systems} \mathrm{for} \mathrm{measuring} \mathrm{bone}$ lead in vivo. Phys Med Biol 1994;39:2263-2271.

81. Hu H, Milder FL, Burger DE. X-Ray fluorescence measurements of lead burden in subjects with low-level community lead exposure. Arch Environ Health 1990;45(6):335-341.

82. Cooper DB. Epidemias por plomo en la Ciudad de México 17611813. México, D.F.: IMSS, Colección Salud y Seguridad Social, Serie Historia, 1980:39.

83. Calderón-Salinas JV, Valdez-Anaya B, Mazúñiga-Charles, Albores-Me$\operatorname{dina} A$. Lead exposure in a population of Mexican children. Human Exp Toxicol 1996;15:305-311.

84. Contreras R. Gasolinas de alto octanaje con bajo contenido de plomo y azufre. Rev Mex Higiene Seguridad 1990: 4-17.

85. Hernández-Avila M. El plomo: un problema de salud pública en México. En: Hernández-Avila M y Palazuelos-Rendón E, ed. Intoxicación por plomo en México: prevención y control. Cuernavaca, Mor.: Instituto $\mathrm{Na}$ cional de Salud Pública, Perspectivas en Salud Pública, núm. 21, 1995:13-24. 86. Montoya-C abrera MA, Maldonado-Torres L, Landazuri-Laris P, Montes-Allende F, Escobar-Márquez R, Margain-C ompean JC. D eterminación de plomo en la sangre del cordón umbilical en recién nacidos normales. Arch Inves Med 1981;12:457-462.

87. Palazuelos-Rendón E. Plomo y salud. Impacto ambiental de la reformulación de las gasolinas en la Zona Metropolitana de la Ciudad de México. Una evaluación económica. En:H ernández-Avila M y Palazuelos-Rendón E. Intoxicación por plomo en México: prevención y control. Cuernavaca, Mor.: Instituto $\mathrm{N}$ acional de Salud Pública, Perspectivas en Salud Pública, núm. 21, 1995:257-288.

88. Kramer MS, $\mathrm{O}$ livier M, McLean FH et al. Impact of intrauterine growth retardation and body proportionality on fetal and neonatal outcome. Pediatrics 1990;85:707-713.

89. A vila-C uriel A, C hávez-Villasana A, Shamah-Levy T, Madrigal-Fritsch H. La desnutrición infantil en el medio rural mexicano: análisis de las encuestas nacionales de alimentación. Salud Publica Mex 1993;35:658-666.

90. Hernández-A vila M, Sanín LH, Romieu I, Palazuelos E, Tapia-C onyer $R, O$ laiz $G$ et al. Higher milk intake during pregnancy is associated with lower maternal and umbilical cord lead levels in post-partum women. Environ Res 1997; 76:116-122.

91 U.S. Department of Health and Human Services. Toxicological profil for lead. A gency for toxic substances and disease registry. Atlanta: Division of Toxicology/Toxicology Information Branch, 1993. 